\title{
Very high energy gamma-ray observation of the peculiar transient event Swift J1644+57 with the MAGIC telescopes and AGILE
}

\author{
J. Aleksić ${ }^{1}$, L. A. Antonelli ${ }^{2, \star}$, P. Antoranz ${ }^{3}$, M. Asensio ${ }^{4}$, M. Backes ${ }^{5}$, U. Barres de Almeida ${ }^{6}$, J. A. Barrio ${ }^{4}$, \\ J. Becerra González ${ }^{7}$, W. Bednarek ${ }^{8}$, K. Berger ${ }^{7,9}$, E. Bernardini ${ }^{10}$, A. Biland ${ }^{11}$, O. Blanch ${ }^{1}$, R. K. Bock ${ }^{6}$, A. Boller ${ }^{11}$, \\ S. Bonnefoy ${ }^{4}$, G. Bonnoli ${ }^{2}$, D. Borla Tridon ${ }^{6}$, T. Bretz ${ }^{12,28}$, E. Carmona ${ }^{13}$, A. Carosi ${ }^{2}$, D. Carreto Fidalgo ${ }^{12,4}$, \\ P. Colin ${ }^{6}$, E. Colombo ${ }^{7}$, J. L. Contreras ${ }^{4}$, J. Cortina ${ }^{1}$, L. Cossio ${ }^{14}$, S. Covino ${ }^{2}$, P. Da Vela ${ }^{3}$, F. Dazzi ${ }^{14,29}$, \\ A. De Angelis ${ }^{14}$, G. De Caneva ${ }^{10}$, B. De Lotto ${ }^{14}$, C. Delgado Mendez ${ }^{13}$, M. Doert ${ }^{5}$, A. Domínguez ${ }^{15}$, D. Dominis
} Prester $^{16}$, D. Dorner ${ }^{12}$, M. Doro ${ }^{17}$, D. Eisenacher ${ }^{12}$, D. Elsaesser ${ }^{12}$, E. Farina ${ }^{18}$, D. Ferenc ${ }^{16}$, M. V. Fonseca ${ }^{4}$, L. Font ${ }^{17}$, C. Fruck $^{6}$, R. J. García López ${ }^{7,9}$, M. Garczarczyk ${ }^{7}$, D. Garrido Terrats ${ }^{17}$, M. Gaug ${ }^{17}$, G. Giavitto ${ }^{1}$, N. Godinović ${ }^{16}$, A. González Muñoz ${ }^{1}$, S. R. Gozzini ${ }^{10}$, A. Hadamek ${ }^{5}$, D. Hadasch ${ }^{19}$, A. Herrero ${ }^{7,9}$, J. Hose ${ }^{6}$, D. Hrupec ${ }^{16}$, W. Idec ${ }^{8}$, F. Jankowski ${ }^{10}$, V. Kadenius ${ }^{20}$, S. Klepser ${ }^{1,30}$, M. L. Knoetig ${ }^{6}$, T. Krähenbühl ${ }^{11}$, J. Krause ${ }^{6}$, J. Kushida ${ }^{21}$, A. La Barbera ${ }^{2}$, D. Lelas ${ }^{16}$, N. Lewandowska ${ }^{12}$, E. Lindfors ${ }^{20,31}$, S. Lombardi $^{2}$, M. López ${ }^{4}$, R. López-Coto ${ }^{1}$,

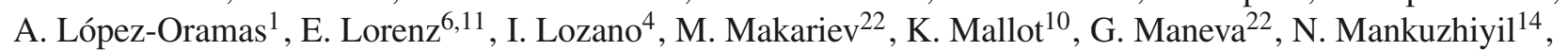
K. Mannheim ${ }^{12}$, L. Maraschi ${ }^{2}$, B. Marcote ${ }^{23}$, M. Mariotti ${ }^{24}$, M. Martínez ${ }^{1}$, J. Masbou ${ }^{24}$, D. Mazin ${ }^{6}$, M. Meucci ${ }^{3}$, J. M. Miranda ${ }^{3}$, R. Mirzoyan ${ }^{6}$, J. Moldón ${ }^{23}$, A. Moralejo ${ }^{1}$, P. Munar-Adrover ${ }^{23}$, D. Nakajima ${ }^{6}$, A. Niedzwiecki ${ }^{8}$, K. Nilsson ${ }^{20,31}$, N. Nowak ${ }^{6}$, R. Orito ${ }^{21}$, S. Paiano ${ }^{24}$, M. Palatiello ${ }^{14}$, D. Paneque ${ }^{6}$, R. Paoletti ${ }^{3}$, J. M. Paredes ${ }^{23}$, S. Partini ${ }^{3}$, M. Persic ${ }^{14,25}$, F. Prada ${ }^{15,32}$, P. G. Prada Moroni ${ }^{26}$, E. Prandini ${ }^{24}$, I. Puljak ${ }^{16}$, I. Reichardt ${ }^{1}$, R. Reinthal ${ }^{20}$, W. Rhode ${ }^{5}$, M. Ribó ${ }^{23}$, J. Rico ${ }^{27,1}$, A. Saggion ${ }^{24}$, K. Saito ${ }^{21}$, T. Y. Saito ${ }^{6}$, M. Salvati ${ }^{2}$, K. Satalecka ${ }^{4}$, V. Scalzotto ${ }^{24}$, V. Scapin ${ }^{4}$, C. Schultz ${ }^{24}$, T. Schweizer ${ }^{6}$, S. N. Shore ${ }^{26}$, A. Sillanpää20 ${ }^{20}$ J. Sitarek ${ }^{1}$, I. Snidaric ${ }^{16}$, D. Sobczynska ${ }^{8}$, F. Spanier ${ }^{12}$, S. Spiro ${ }^{2}$, V. Stamatescu ${ }^{1}$, A. Stamerra ${ }^{3}$, B. Steinke ${ }^{6}$, J. Storz ${ }^{12}$, S. Sun ${ }^{6}$, T. Surić ${ }^{16}$, L. Takalo ${ }^{20}$,

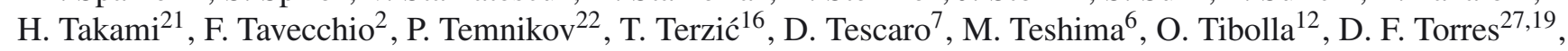
T. Toyama ${ }^{6}$, A. Treves ${ }^{18}$, M. Uellenbeck ${ }^{5}$, P. Vogler ${ }^{11}$, R. M. Wagner ${ }^{6}$, Q. Weitzel ${ }^{11}$, F. Zandanel ${ }^{15}$, R. Zanin ${ }^{23}$, (The MAGIC Collaboration), F. Longo ${ }^{33}$, F. Lucarelli ${ }^{34}$, C. Pittori ${ }^{34}$, and F. Verrecchia ${ }^{34}$ for the AGILE team (Affiliations can be found after the references)

Received 30 January 2013 / Accepted 15 February 2013

\section{ABSTRACT}

Context. On March 28, 2011, the BAT instrument on board the Swift satellite detected a new transient event that in the very beginning was classified as a gamma ray burst (GRB). However, the unusual X-ray flaring activity observed from a few hours up to days after the onset of the event made a different nature seem to be more likely. The long-lasting activity in the X-ray band, followed by a delayed brightening of the source in infrared and radio activity, suggested that it is better interpreted as a tidal disruption event that triggered a dormant black hole in the nucleus of the host galaxy and generated an outflowing jet of relativistic matter.

Aims. Detecting a very high energy emission component from such a peculiar object would be enable us to constrain the dynamic of the emission processes and the jet model by providing information on the Doppler factor of the relativistic ejecta .

Methods. The MAGIC telescopes observed the peculiar source Swift J1644+57 during the flaring phase, searching for gamma-ray emission at very-high energy (VHE, $E>100 \mathrm{GeV}$ ), starting observations nearly 2.5 days after the trigger time. MAGIC collected a total of $28 \mathrm{~h}$ of data during 12 nights. The source was observed in wobble mode during dark time at a mean zenith angle of $35^{\circ}$. Data were reduced using a new image-cleaning algorithm, the so-called sum-cleaning, which guarantees a better noise suppression and a lower energy threshold than the standard analysis procedure.

Results. No clear evidence for emission above the energy threshold of $100 \mathrm{GeV}$ was found. MAGIC observations permit one to constrain the emission from the source down to $100 \mathrm{GeV}$, which favors models that explain the observed lower energy variable emission. Data analysis of simultaneous observations from AGILE, Fermi and VERITAS also provide negative detection, which additionally constrain the self-Compton emission component.

Key words. radiation mechanisms: non-thermal - galaxies: active - gamma rays: general

^ Corresponding Authors:

e-mail: [angelo.antonelli; alessandro.carosi;

saverio.lombardi]@oa-roma.inaf.it

e-mail: ulisses@mppmu.mpg.de

e-mail: stefano.covino@brera.inaf.it

\section{Introduction}

It is widely accepted that almost every galaxy hosts a massive black hole (MBH) in its core. The majority of these are believed to be in a dormant state and only a subclass of galaxies show evidence for an active nucleus. The continuous gas accretion onto the MBH is the primary source of the observed emission 
in these active galactic nuclei (AGN). The existence of MBHs at the center of non-active galaxies can be inferred in different ways; one of these is the occasional tidal disruption of stars and the subsequent accretion of their debris by MBHs (see e.g. Hills 1975; Rees 1988; Komossa 2002). When fueled by such a tidal disruption event (TDE), black hole that had been quiescent until then can suddenly enter a violent period of flaring emission. These events have been theoretically predicted in the past (see e.g. Hills 1975; Rees 1988) and observations of flaring emission from usually non-active galaxies, interpreted as possible TDEs, have previously been reported in the literature (see e.g. Komossa 2002; Halpern et al. 2004; Maksym et al. 2010). Tidal disruption events may be quite common in the Universe at different scales: Campana et al. (2011b) have recently reported on a possible tidal disruption of a small body (of comet or asteroid mass-scale) on a neutron star related to the GRB-like event GRB 101225.

In this paper we report on the VHE observations of the Swift J1644+57 (a.k.a. Sw 1644+57) transient event with the MAGIC telescopes. This unusual event has been interpreted as the outburst emission from an $\mathrm{MBH}$ triggered by the accretion of a tidally disrupted star. Evidence for a highly variable relativistic jet-beamed outflow has also been observed, leading to a clear analogy with a blazar-like object (Barres de Almeida \& De Angelis 2011; Bloom et al. 2011; Burrows et al. 2011).

In Sect. 2, we briefly describe the multiwavelegth behavior of the transient event. In Sects. 3-5 we describe MAGIC and AGILE follow-up observations and data analysis, while Sects. 6 and 7 present the main results and their interpretative scenario. Throughout this paper the convention for a generic quantity $Q_{x}=Q / 10^{x}$ was adopted.

\section{The Sw $1644+57$ transient event}

The Sw 1644+57 transient was first detected on March 28, 2011 at 12:57:45 UT by the Burst Alert Telescope (BAT, 15-150 keV) on board the Swift satellite, and was classified as a GRB event (GRB110328A, Cummings et al. 2011). The X-Ray Telescope (XRT) on board the Swift satellite started to observe the field about $1474 \mathrm{~s}$ after the BAT trigger, finding a bright, uncataloged X-ray source at coordinates $\operatorname{RA}(\mathrm{J} 2000)=16^{\mathrm{h}} 44^{\mathrm{m}} 49.29^{\mathrm{s}}, \operatorname{Dec}(\mathrm{J} 2000)=+57^{\circ} 34^{\prime} 50.8^{\prime \prime} . A$ GRB nature of the event was rapidly ruled out because a very fast flaring activity $\left(t_{\mathrm{var}} \sim 100 \mathrm{~s}\right.$ time scale) was detected, with strong flux variations (about a factor of few hundreds), and it lasted for several hours, which is larger than the typical time scale of a GRB. Because of this flaring activity, Swift-BAT was re-triggered three times after the first outburst, on March 28 at 13:40:41 UT and on March 29 at 18:26:25 UT and 19:57:45 UT (Barthelmy et al. 2011; Sakamoto et al. 2011). This unusual behavior prompted several multiwavelength follow-up observations. An optical counterpart of the X-ray transient was detected by the Nordic Optical Telescope (NOT; Leloudas et al. 2011), which observed the field in the BVR filters at 01:47 UT (i.e., 12.8 and $12.1 \mathrm{~h}$ after the first and the second trigger, respectively) and detected an object at coordinates consistent with the enhanced Swift-XRT error circle. The source was also detected at near-IR wavelengths (Morgan et al. 2011; Levan et al. 2011c) by groundbased telescopes and by the Hubble Space Telescope (HST) at both the near-IR and optical ( $F 160 \mathrm{~W}$ and $F 606 \mathrm{~W}$ filters respectively). The HST detected a point-like IR source consistent with the X-ray position of the transient event. Optical band revealed a galaxy whose nucleus was consistent with the position of the IR source (Fruchter et al. 2011). Evidence of variability was observed only in the IR band (Levan et al. 2011b), whereas the source did not show any measurable luminosity changes in the optical range (Leloudas et al. 2011). Owing to the relatively high $\mathrm{NH}$ value needed to fit X-ray spectra $(\mathrm{NH} \sim$ $2 \times 10^{22} \mathrm{~cm}^{-2}-$ Burrows et al. 2011), the optical counterpart is thought to be heavily obscured, implying that any optical emission from the transient event was overwhelmed by the non variable host galaxy contribution. Levan et al. (2011a) performed spectroscopic observations of the optical source with Gemini/GMOS on March 29, 2011, showing $\mathrm{H}_{\beta}$ and $\mathrm{O}_{\text {III }}$ emission lines (4959 and $5007 \AA$, respectively) at a common redshift of $z=0.353$. This measurement was rapidly confirmed by independent observations with the Gran Telescopio Canarias (GTC - Thöne et al. 2011). At lower energies, several radio detections were also reported: Zauderer et al. (2011a) observed with the Expanded Very Large Array (EVLA) at two frequencies (1 GHz bandwidth each) centered at 4.94 and $6.69 \mathrm{GHz}$. They found a single, unresolved radio source within the SwiftXRT error circle that is coincident with the optical source, which maintained radio activities up to two days after the initial trigger (Zauderer et al. 2011b). This provided the first clear evidence that connected the quiescent optical source, which is positionally coincident with the brightening radio source, with the transient $\mathrm{X}$-ray/gamma-ray source.

The sky region of the transient was also observed by the Fermi-LAT instrument in the $100 \mathrm{MeV}-10 \mathrm{GeV}$ energy range and by AGILE above $50 \mathrm{MeV}$. At the time of the first source trigger, Fermi was operating in pointing mode and during the first $\sim 2.25 \mathrm{~h}$ the source was at $47^{\circ}$ from the LAT boresight. No significant gamma-ray emission has been detected from the transient on daily timescales or on shorter (about $1 \mathrm{~h}$ around each trigger) timescales. Moreover, negative detection was also obtained from the analysis of the whole Fermi-LAT database (Omodei et al. 2011). A dedicated AGILE maximum-likelihood analysis on three different timescales did not yield any detection above $4 \sigma$. In the very high energy regime (>100 GeV), the VERITAS Cherenkov telescopes performed observations from March 292011 starting $22.5 \mathrm{~h}$ after the first BAT trigger and they continued to monitor the source for $\sim 2 \mathrm{~h} /$ night until April 15 2011. No gamma-ray emission was detected from the direction of the source in the complete data set or on shorter timescales, and upper limits were derived at $\sim 500 \mathrm{GeV}$ (Aliu et al. 2011).

\section{MAGIC observations and analysis techniques}

The MAGIC system consists of two $17 \mathrm{~m}$ dish Imaging Atmospheric Cherenkov Telescopes (IACTs) located at the Roque de los Muchachos observatory $\left(28.8^{\circ} \mathrm{N}, 17.8^{\circ} \mathrm{W}, 2200 \mathrm{~m}\right.$ a.s.1.), on the Canary Island of La Palma. Since late 2009 the MAGIC system is carrying out stereoscopic observations with a sensitivity of $<0.8 \%$ of the Crab Nebula flux for energies above $\sim 300 \mathrm{GeV}$ in $50 \mathrm{~h}$ of observations, and a trigger energy threshold of $50 \mathrm{GeV}$ (Aleksić et al. 2011a). MAGIC performed a follow-up measurement of Sw J1644+57, starting observations on March 312011 at 02:24 UT, approximately 2.5 days after the first BAT trigger. MAGIC continued to observe the source in the subsequent days up to April 15 2011, when follow-up was interrupted because the Moon was almost full (see Fig. 1). A total effective time of about $28 \mathrm{~h}$ of observations have been collected for this event with a zenith angle ranging from $28^{\circ}$ to $47^{\circ}$, which resulted in an analysis energy threshold (defined as the peak of the simulated energy distribution for a Crab-Nebula-like spectrum after cuts) of $100 \mathrm{GeV}$. The data analysis was performed using the standard software package 


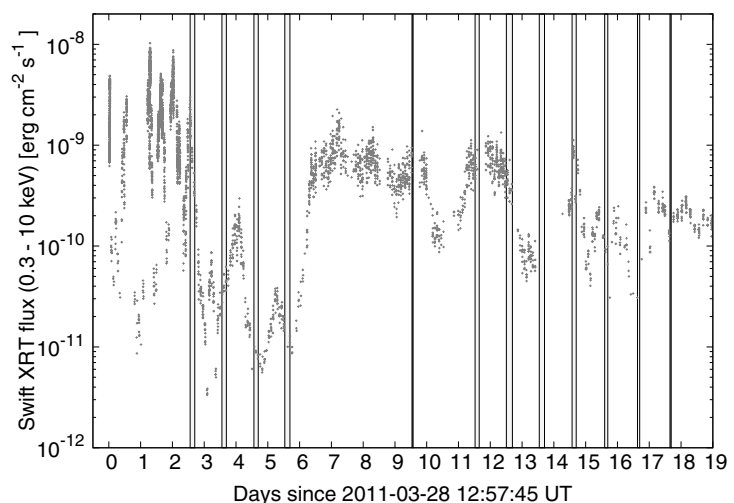

Fig. 1. MAGIC observation windows of the Sw J1644+57 transient from March 31 to April 15 2011, superimposed on the X-ray light curve from Swift-XRT. A time-dependent conversion factor has been used to convert the count rate into physical units as specified in Burrows et al. (2011).

MARS (Albert et al. 2008a; Aliu et al. 2009), including newly developed tools for the stereoscopic data reduction (Moralejo et al. 2009; Lombardi et al. 2011; Aleksić et al. 2011a). After calibrating the events recorded by each telescope (Albert et al. 2008 b), the data were processed with the so-called sum-cleaning algorithm (Lombardi et al. 2011), which has been proven to perform better than the standard cleaning method (Aliu et al. 2009; Aleksić et al. 2011a) because it suppresses the night-sky background and electronic noise better, and has a lower energy threshold (Zanin et al. 2011). The input threshold levels for the sum-cleaning method were set for MAGIC-I (MAGIC-II) ${ }^{1}$ data to 4 (7) photo-electrons (phes) for the core pixels (belonging to the shower images), and 3 (4) phes for the boundary pixels ${ }^{2}$. For the gamma/hadron separation and gamma-direction estimation a multivariate method based on a random forest (RF) algorithm was applied (Albert et al. 2008c). This algorithm employs some Cherenkov image parameters (Hillas 1985) to compute a gamma/hadron discriminator called Hadronness by comparison with Monte Carlo gamma-ray simulations. The energy of the events was estimated by averaging individual energy estimators for both telescopes based on look-up tables (Aleksić et al. 2011a).

After applying standard quality checks based on the rate of the stereo events and the distributions of basic image parameters, the selected data sample from which we derived the results presented here consisted of $27.4 \mathrm{~h}$ of effective observation time. The rejected data were affected mainly by poor atmospheric conditions during data taking. The final analysis cuts applied to Sw J1644+57 data were optimized on Crab Nebula data and MC simulations (Aleksić et al. 2011a). In computing the significance of the signal coming from the transient object, single cuts in Hadronness and $\theta^{2}$ (see Sect. 4) optimized for energies close to the threshold were applied. Conversely, while deriving upper

\footnotetext{
1 We refer to MAGIC-I as the first-built MAGIC telescope that operated in stand-alone mode until 2009 while MAGIC-II is the second newer instrument.

2 The sum-cleaning setting applied to MAGIC-II data has threshold values different from MAGIC-I because of the intrinsic differences between the photo-multipliers (PMT) of the two cameras and readouts of the two MAGIC telescopes (Cortina et al. 2009). The levels are optimized separately for each telescope's data to maximize the noise suppression and, at the same time, to lower the achievable energy threshold (Lombardi et al. 2011; Zanin et al. 2011).
}

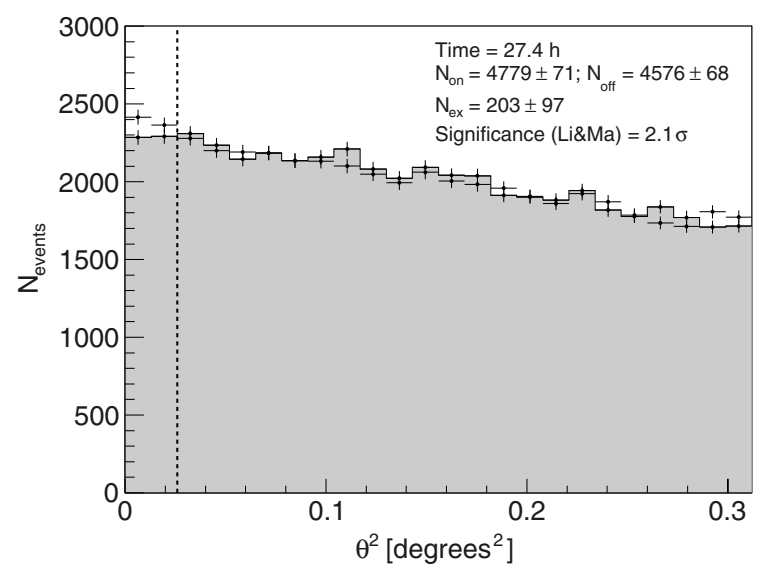

Fig. 2. $\theta^{2}$ distributions for $\mathrm{Sw} \mathrm{J} 1644+57$ signal and background estimation from $27.4 \mathrm{~h}$ of MAGIC stereo observations taken between March 31 and April 152011 above an energy threshold of $100 \mathrm{GeV}$. The region between zero and the vertical dashed line (at $0.026 \mathrm{deg}^{2}$ ) represents the signal region.

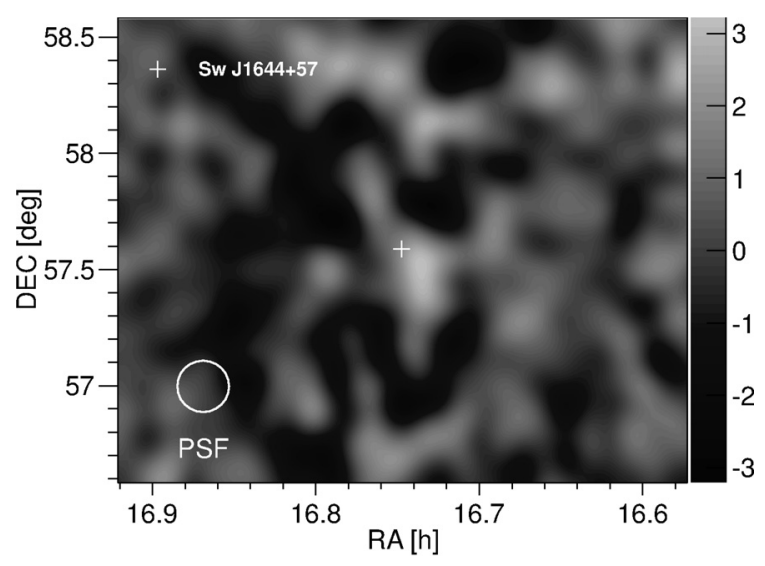

Fig. 3. Significance skymap of the sky region around Sw J1644+57 from $27.4 \mathrm{~h}$ of MAGIC stereo observations taken between March 31 and April 15 2011, above an energy threshold of $100 \mathrm{GeV}$. The position of Sw J1644+57 is marked with a white cross. The colours palette indicates the significance values while the point spread function (PSF) of about $0.12^{\circ}$ is also displayed.

limits (ULs) on the flux of the source, multiple cuts optimized in logarithmic energy bins were considered.

\section{Results}

The $\theta^{2}$ plot (i.e., the distributions of the square angular distance between the reconstructed source and the nominal positions of the signal and background control regions) for energies above $100 \mathrm{GeV}$ is shown in Fig. 2. We found an excess of $203 \pm 97$ events in the fiducial signal region with $\theta^{2}<$ $0.026 \mathrm{deg}^{2}$, corresponding to a significance of 2.1 standard deviations $(\sigma)$, calculated according to Eq. (17) of Li \& Ma (1983).

The significance skymap above $100 \mathrm{GeV}$ of the sky region around Sw J1644+57 is shown in Fig. 3. The significance distribution is consistent with background fluctuations. Similar results were obtained on a daily-scale analysis performed up to 18 days after trigger time.

Because no significant signal over the background was found in the data, we derived ULs on the flux of the source, as explained in Aleksić et al. (2011b). Table 1 summarizes the differential ULs obtained in three energy logarithmic bins 
Table 1. Differential flux ULs $\phi^{\mathrm{UL}}$ in three energy logarithmic bins between $100 \mathrm{GeV}$ and $1 \mathrm{TeV}$, assuming a power-law spectrum with spectral index of $\Gamma=-3$.

\begin{tabular}{|c|c|c|c|c|}
\hline $\begin{array}{l}\text { Energy bin } \\
{[\mathrm{GeV}]}\end{array}$ & $\begin{array}{c}\langle E\rangle \\
{[\mathrm{GeV}]}\end{array}$ & $\begin{array}{c}N_{\mathrm{ON}} / N_{\mathrm{OFF}} \\
\left(\sigma_{\mathrm{LiMa}}\right)\end{array}$ & $N_{\mathrm{ex}}^{\mathrm{UL}}$ & $\begin{array}{c}\phi^{\mathrm{UL}} \\
{\left[\mathrm{TeV}^{-1} \mathrm{~cm}^{-2} \mathrm{~s}^{-1}\right.}\end{array}$ \\
\hline $100-250$ & 145 & $\begin{array}{c}5362 / 5146 \\
(2.1)\end{array}$ & 1124 & $3.5 \times 10^{-10}$ \\
\hline $250-500$ & 322 & $\begin{array}{c}370 / 316 \\
(2.1)\end{array}$ & 283 & $2.1 \times 10^{-11}$ \\
\hline 500-1000 & 651 & $\begin{array}{l}87 / 90 \\
(-0.2)\end{array}$ & 49 & $1.2 \times 10^{-12}$ \\
\hline
\end{tabular}

Notes. For each energy bin, the pivot energy and the number of events in the signal region $\left(N_{\mathrm{ON}}\right)$ and background control region $\left(N_{\mathrm{OFF}}\right)$ are also shown, together with the corresponding significance $\sigma_{\mathrm{LiMa}}$ and UL on the number of excess events $N_{\mathrm{ex}}^{\mathrm{UL}}$ at $99 \% \mathrm{CL}$.

between $100 \mathrm{GeV}$ and $1 \mathrm{TeV}$, assuming a generic power-law gamma-ray spectrum with spectral index of $\Gamma=-3$ and using the method of Rolke et al. (2005), with a confidence level (CL) of $99 \%$ and a total systematic uncertainty of $30 \%$. To investigate the ULs sensitivity to the assumed photon index, we also evaluated differential limits using $\Gamma=-2$ and $\Gamma=-2.5$. We did not find significant differences in UL values, and the highest difference was about $15 \%$, which constrains the limit in the first energy bin using $\Gamma=-2.5$. These differences are still within our uncertainties for the effective area evaluation.

Figure 4 shows the spectral energy distribution (SED) of Sw J1644+57 as measured by different instruments from radioto gamma-ray wavelengths. The experimental ULs achieved from the MAGIC observation (black arrows in the plot) represent the most stringent constraints at VHE down to $100 \mathrm{GeV}$ currently available.

\section{AGILE-GRID data analysis}

The AGILE-GRID instrument on board the AGILE satellite is a compact gamma-ray imaging telescope that operates in the $30 \mathrm{MeV}-50 \mathrm{GeV}$ energy range (Tavani et al. 2009). The AGILEGRID automatic procedure used to detect emission from GRB localized in hard X-rays by other instruments (see Moretti et al. 2009 , for a description of the technique) did not find evidence for excesses over background in a $100 \mathrm{~s}$ time interval following the Swift-BAT triggers on the event. To derive estimated flux or flux upper limits of the source, we ran the AGILE point source analysis software, which is based on the maximum-likelihood (ML) technique, using a radius of analysis of $10^{\circ}$. AGILE-GRID data processed using the AGILE standard analysis pipeline at the AGILE Data Center (Pittori et al. 2009) were analyzed with the latest scientific software (AGILE_SW_5.0_SourceCode) and inflight calibrations (I0023) publicly available since 30-09-2011 at the ASDC website. Standard photon selections were applied to events. Events collected during passages of the South Atlantic Anomaly or whose reconstructed directions form angles with the satellite-Earth vector smaller than $80^{\circ}$ were rejected to avoid Earth-albedo contamination. Counts, exposure, and galactic background gamma-ray maps were created with a bin-size of $0.5^{\circ} \times 0.5^{\circ}$ for $E>100 \mathrm{MeV}$, including all events collected up to $60^{\circ}$ off-axis. The ML analysis above $100 \mathrm{MeV}$ was performed over three timescales: two days from 2011 March 28, five days from 2011 March 28 and a long 46-day interval starting on March 15, 2011.

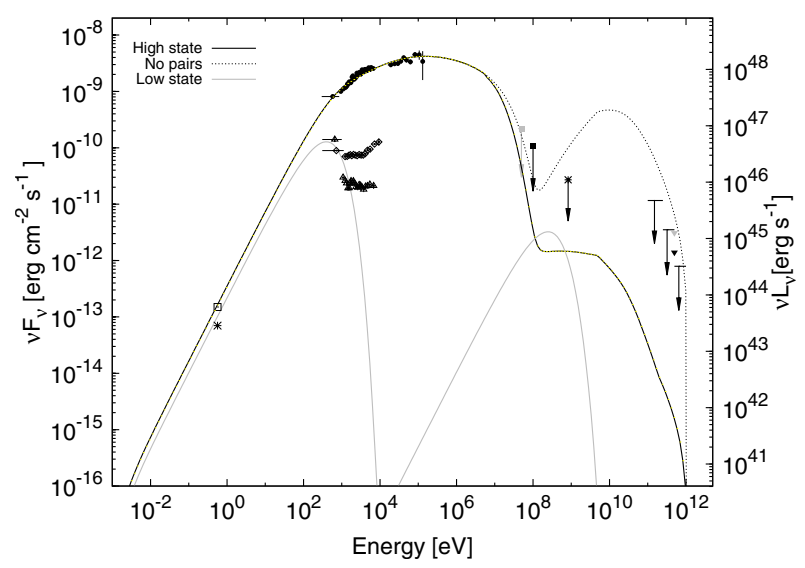

Fig. 4. Spectral energy distribution of the Sw J1644+57 event as modeled in Burrows et al. (2011). The black curve is the modeled SED around the peak of the X-ray flares ( $\sim 31 \mathrm{~h}$ after event onset) with and without flux suppression by pair production (solid and dashed lines, respectively). The gray curve shows the corresponding model in the low $\mathrm{X}$-ray flux state at $\sim 5.5$ days after the trigger. Black circle data points are the XRT and BAT fluxes as measured few days after the BAT trigger; diamond points show the measured SED at 4.5 days, while triangle data points are XRT data between 6.5 and 9.5 days after the BAT trigger. The black arrows represent the ULs derived from the MAGIC observations that are presented in this work (Table 1). The down-pointing black and gray triangles in the VHE range are the VERITAS UL observations for the total and flaring period. In the HE range, AGILE upper limits in the 50-200 MeV (gray square) and $>100 \mathrm{MeV}$ (black square) are reported for an integration time of 5 and 2 days (this work). The Fermi-LAT limit between March 28 to April 4 (star UL) is also reported. In the optical range, $K$-band data form the Telescopio Nazionale Galileo (TNG) from about 2.5 days (open square) and 4.5 days (star) after the onset are shown.

We obtained no detection above the $3 \sigma$ significance level from the position of the X-ray transient. The low statistical significance does not allow a positive detection of the source and limits the AGILE spectral analysis capability with the chosen data sample. The standard practice procedure in similar cases is to assume a typical spectral photon index of 2.1 for the likelihood analysis. This assumption is motivated by the known Crablike spectral properties of the majority of AGILE gamma-ray sources above $100 \mathrm{MeV}$. Because of the low-energy resolution of AGILE (on the order of $100 \%$ at $300 \mathrm{MeV}$ ), and crosstalk among different energy channels, the integral flux UL were computed by taking into account all gamma-rays above $100 \mathrm{MeV}$ without energy spectral deconvolution. In cases with low statistics like these, taking into account the instrument performance over daily-to-monthly timescales in extragalactic sky regions, the AGILE Team also suggest to scale the corresponding differential $v F(v)$ values to be shown in spectral energy distributions around $\sim 100 \mathrm{MeV}$, which corresponds to the lower end of the energy bins considered, where the AGILE-GRID sensitivity is best.

The AGILE 95\% c.l. flux UL thus obtained above $100 \mathrm{MeV}$ are shown in Table 2 together with the corresponding differential $v F(v)$ values scaled at the lower end of the energy bins considered.

We also performed a dedicated analysis in the softer 50-200 MeV AGILE energy band, with the same event selections and map parameters as above, on the 5- and 46-days time intervals. The resulting 95\% flux UL, scaled at the $50 \mathrm{MeV}$ energy point, are also shown in Table 2. 
Table 2. Flux upper limits (UL at $95 \%$ c. 1.) from the AGILE-GRID data analysis.

\begin{tabular}{lccc}
\hline \hline $\begin{array}{c}\text { Integration } \\
\text { [days] }\end{array}$ & $\begin{array}{c}\text { Energy bin } \\
{[\mathrm{MeV}]}\end{array}$ & $\begin{array}{c}\text { Flux (UL) } \\
{\left[\mathrm{ph} \mathrm{cm}^{-2} \mathrm{~s}^{-1}\right]}\end{array}$ & $\begin{array}{c}v F(v) \\
\left.\mathrm{erg} \mathrm{cm}^{-2} \mathrm{~s}^{-1}\right]\end{array}$ \\
\hline $2^{a}$ & $>100$ & $6.2 \times 10^{-7}$ & $1.1 \times 10^{-10}$ \\
$5^{a}$ & $50-200$ & $1.9 \times 10^{-6}$ & $2.1 \times 10^{-10}$ \\
$5^{a}$ & $>100$ & $3.6 \times 10^{-7}$ & $6.3 \times 10^{-11}$ \\
$46^{b}$ & $>100$ & $1.4 \times 10^{-7}$ & $2.5 \times 10^{-11}$ \\
$46^{b}$ & $50-200$ & $0.5 \times 10^{-6}$ & $0.6 \times 10^{-10}$ \\
\hline
\end{tabular}

Notes. ${ }^{(a)}$ Days from 2011 March 28. ${ }^{(b)}$ Days from 2011 March 15.

\section{Discussion}

Using the mass-luminosity relationship described in Graham (2007), Barres de Almeida \& De Angelis (2011) derived an upper limit of about a few $10^{7} M_{\odot}$ to the object mass. This estimate is incompatible with the observed X-ray peak luminosity of $\sim 5 \times 10^{48} \mathrm{erg} / \mathrm{s}$, which requires a higher mass limit to the hypothesized accretion in order not to exceed the Eddington limit ( $M \gtrsim 3 \times 10^{10} M_{\odot}$, Campana et al. 2011a). The difference can be explained by interpreting the variable emission as coming from relativistic jets of outflowing material. The hypothesis of beamed jets-emission (Campana et al. 2011a) implies a natural interpretation of the event as a new-born small-scale blazar triggered by the TDE (Barres de Almeida \& De Angelis 2011; Burrows et al. 2011; Bloom et al. 2011). In this context, Burrows et al. (2011) used the synchrotron self Compton (SSC) mechanism to model the observed emission.

However, the $v^{1 / 3}$ slope between the optical and the X-ray bands suggests that the observed radiation could be synchrotronemission originated from an electron population with a relatively high low-energy cut-off (Katarzyński et al. 2005). In contrast to a standard blazar modeling, this implies a very limited amount of matter in the jet and, according to this scenario, a magnetic-dominated jet with a synchrotron component peaking in the X-ray band has to be assumed. At higher energies, the negative detection from AGILE, Fermi-LAT, VERITAS, and MAGIC strongly constrained the self-Compton emission component, although it is difficult to compare upper limits obtained at different epochs. On the other hand, the temporal evolution of the VHE emission strictly depends on the model details. The early (a couple of days) emission is sustained by the innermost bound debris from the disrupted star. However, according to Barres de Almeida \& De Angelis (2011), for instance, an extend debris disk will be formed and accretion from this material will be less intense following the viscous time-scale of the disk. It is difficult to evaluate this timescale but it is likely responsible for the long-term emission recorded for months after the main event at lower energies. The absence of gamma-rays is likely due to the compactness of the source and consequent pair-creation opacity. At the same time, the negative detection on daily timescale observations shows that no new enhanced IC component was added to the event on later dates.

Very high energy photons in the MAGIC band $\left(E_{\mathrm{VHE}}^{\prime}\right)$ can be absorbed by a target photon of energy $E_{\mathrm{t}}^{\prime}=\frac{\left(m_{\mathrm{e}} c^{2}\right)^{2}}{E_{\mathrm{VHE}}^{\prime}}$ (primed quantities are expressed in the comoving frame). Assuming, for events close to threshold, a $\gamma \gamma$ cross section of $\sim \frac{\sigma_{\mathrm{T}}}{5}$, where $\sigma_{\mathrm{T}}$ is the Thomson cross section (Svensson 1987), this implies an optical depth for photon-photon interaction $\tau_{\gamma \gamma}^{\text {int }}$

$\tau_{\gamma \gamma}^{\mathrm{int}}\left(E_{\mathrm{VHE}}^{\prime}\right)=\sigma_{\gamma \gamma} n^{\prime}\left(E_{\mathrm{t}}^{\prime}\right) E_{\mathrm{t}}^{\prime} R^{\prime} \simeq \frac{\sigma_{\mathrm{T}}}{5} \frac{L^{\prime}\left(E_{\mathrm{t}}^{\prime}\right)}{4 \pi R^{\prime} c E_{\mathrm{t}}^{\prime}}$, where $n^{\prime}\left(E_{\mathrm{t}}^{\prime}\right)$ is the target photon number density per unit of energy, $L^{\prime}\left(E_{\mathrm{t}}^{\prime}\right)$ is the source luminosity at $E_{\mathrm{t}}^{\prime}$ and $R \sim c t_{\mathrm{var}} \frac{\delta}{1+z}$ is the length path. Following Dondi \& Ghisellini (1995), we thus obtain in the observer frame and for a power-law luminosity of the form $L\left(E_{\mathrm{t}}\right) \propto E^{-\alpha}$

$\tau_{\gamma \gamma}^{\mathrm{int}}=(1+z)^{2 \alpha} \delta^{-(4+2 \alpha)} \frac{\sigma_{\mathrm{T}}}{20 \pi h c^{2}} \frac{L\left(E_{\mathrm{t}}\right)}{t_{\mathrm{var}}} \approx 10^{4} \frac{v_{\mathrm{t}}^{1 / 3}}{t_{\mathrm{var}}} \delta^{-10 / 3}$,

where we used $v_{\mathrm{t}}=E_{\mathrm{t}} / h \approx 6 \times 10^{14} \mathrm{~Hz}(\sim 2.6 \mathrm{eV}$, i.e in the UV band) as the frequency of the target photons for $100 \mathrm{GeV}$ gamma rays, $\sim 3.5 \times 10^{44} \mathrm{erg} / \mathrm{s}$ as the corresponding luminosity (see Fig. 4), $\alpha=-1 / 3$ as the slope for the target photons and $z=$ 0.35 . The suppression factor of the intrinsic flux generated by the internal pair production is given by $F_{\text {int }}\left(1-\mathrm{e}^{-\tau_{\gamma \gamma}}\right) / \tau_{\gamma \gamma} \approx F_{\text {int }} / \tau_{\gamma \gamma}$ assuming $\tau_{\gamma \gamma} \gg 1$. The MAGIC upper limit at $\sim 100 \mathrm{GeV}$ is about a factor 10 below the SSC emission component (if there is no internal absorption), which implies an opacity condition at this energy given by $\tau_{\gamma \gamma} \gtrsim 10$. From Eq. (2), a limit on the Doppler factor of the emitting region can be derived as

$\delta \lesssim 50 \times\left(\frac{v_{t, 14}^{1 / 3}}{t_{\mathrm{var}, 2}}\right)^{3 / 10}$,

which gives $\delta \lesssim 60$ using our data.

This upper limit on the Doppler factor is similar to the limit that was used in Burrows et al. (2011) $(\delta \lesssim 20)$. Thus the MAGIC observations confirms the absence of gamma-ray emission from the event toward the $\sim 100 \mathrm{GeV}$ domain, and reinforces the assumption made by Burrows et al. (2011) that the Doppler factor is lower than a few tens. In addition, SSC emission in the MAGIC band likely falls in the so-called Klein-Nishina (KN) regime, where inverse Compton scattering is relatively inefficient.

On the other hand, because of the relatively high redshift of the source, even the extragalactic background light (EBL) absorption could significantly influence the VHE expected spectrum. According to Domínguez et al. (2011), for a $z=0.35$ source, the $\gamma-\gamma$ opacity $\left(\tau_{\gamma \gamma}^{\mathrm{EBL}}\right)$ begins to become important at $\sim 300 \mathrm{GeV}$ (where $\tau_{\gamma \gamma}^{\mathrm{EBL}} \approx 1$ ). This makes the achievable MAGIC energy threshold relevant so that at least the first energy bin is not affected by strong EBL attenuation. A reduction in the intrinsic SSC flux $\left(v F_{v}^{\text {int }}\right)$ of about $25 \%, 70 \%$, and $95 \%$ in each energy bins respectively has also to be taken into account so that the complete expected SSC flux $v F_{v}^{\mathrm{obs}} \approx v F_{\gamma}^{\mathrm{int}} \exp \left[-\left(\tau_{\gamma \gamma}^{\mathrm{EBL}}\right)\right] / \tau_{\gamma \gamma}^{\mathrm{int}}$ is significantly reduced by the two absorptions (internal + EBL) and the $\mathrm{KN}$ effect.

\section{Conclusions}

The MAGIC observation of the unusual Sw J1644+57 transient extends the VHE coverage on this object down to the $\sim 100 \mathrm{GeV}$ energy range. Provided upper limits are compatible with the proposed emission models based on previously published data. The jet model that gives the best fit to the SED data in Burrows et al. (2011) is constrained by the AGILE, Fermi-LAT, VERITAS, and MAGIC upper limits with an IC component that is likely strongly suppressed by $\gamma-\gamma$ pair production. This in turn implies a lower limit on the density of X-ray and optical photons, i.e., an upper limit to the Doppler factor $\delta$, in the X-ray and optical emitting regions. The broad upper limit on the Doppler factor, $\delta \leq 20$ proposed in Burrows et al. (2011) is not significantly tightened 
by the MAGIC results. This value is also consistent with the estimated fraction of TDEs with jets pointed toward us (opening angle of $\theta \sim 5^{\circ}$ ) based on the observed frequency of such events during the lifetime of the orbiting Swift-BAT instrument (Bloom et al. 2011). A suitably high Lorentz factor would allow for a negligible rate of $\gamma \gamma \rightarrow \mathrm{e}^{+} \mathrm{e}^{-}$interaction, which would let the IC emission emerge unscathed. In addition, at these photon energies the IC emission decreases steeply, in the same way that it is likely produced in the $\mathrm{KN}$ regime. For typical jet models, the corresponding highest energy of the electron scattering in the KN regime is $\gamma_{\mathrm{e}, \max } \geq 10^{6}$ (Mankuzhiyil et al. 2011), which additionally reduces the expected flux in the VHE range.

Acknowledgements. We would like to thank Gabriele Ghisellini for providing the SED model points used to describe the object. We would like to thank the Instituto de Astrofísica de Canarias for the excellent working conditions at the Observatorio del Roque de los Muchachos in La Palma. The support of the German BMBF and MPG, the Italian INFN, the Swiss National Fund SNF, and the Spanish MICINN is gratefully acknowledged. This work was also supported by the CPAN CSD2007-00042 and MultiDark CSD2009-00064 projects of the Spanish Consolider-Ingenio 2010 programme, by grant DO02-353 of the Bulgarian NSF, by grant 127740 of the Academy of Finland, by the DFG Cluster of Excellence "Origin and Structure of the Universe", by the DFG Collaborative Research Centers SFB823/C4 and SFB876/C3, and by the Polish MNiSzW grant 745/N-HESS-MAGIC/2010/0.

\section{References}

Albert, J., Aliu, E., Anderhub, H., et al. 2008a, ApJ, 674, 1037

Albert, J., Aliu, E., Anderhub, H., et al. 2008b, Nucl. Instrum. Meth., 594, A407 Albert, J., Aliu, E., Anderhub, H., et al. 2008c, Nucl. Instrum. Meth., 588, A424 Aleksić, J., Alvarez, E. A., Antonelli, L. A., et al. 2011a, Astropart. Phys., submitted [arXiv: 1108 . 1477]

Aleksić, J., Alvarez, E. A., Antonelli, L. A., et al. 2011b, JCAP, 1106, 035

Aliu, E., Anderhub, H., Antonelli, L. A., et al. 2009, Astropart. Phys., 30, 293

Aliu, E., Arlen, T., Aune, T., et al. 2011, ApJ, 738, L30

Barres de Almeida, U., \& De Angelis, A. 2011, A\&A, unpublished [arXiv: 1104.2528B]

Barthelmy, S. D., Baumgartner, W. H., Beardmore, A. P., et al. 2011, GCN, 11824

Bloom, J. S., Giannios, D., Metzger, B. D., et al. 2011, Science, 333, 203

Bulgarelli, A., et al. 2009, GRID Scientific Analysis User Manual, AGILE-IFCOP-009 available on-line at http://agile.asdc.asi .it/public

Burrows, D. N., Kennea, J. A., Ghisellini, G., et al. 2011, Nature, 476, 421B

Camenzind, M., \& Krockenberger, M. 1992, A\&A, 255, 59

Campana, S., Covino, S., Tagliaferri, G., et al. 2011a, GCN, 11843

Campana, S., Lodato, G., D’Avanzo, P., et al. 2011b, Nature, 480, 69

Cattaneo, P. W., Argan, A., Boffelli, F., et al. 2011, NIM A, 630, 251

Cavallo, G., \& Rees, M. J. 1978, MNRAS, 183, 359

Cortina, J., Goebel, F., Schweizer, T., et al. 2009, Proc. of 31st ICRC, Łódź, Poland [arXiv:0907.1211]

Cummings, J. R., Barthelmy, S. B., Beardmore, A. P., et al. 2011, GCN, 11823 Domínguez, A., Primack, J. R., Rosario, D. J., et al. 2011, MNRAS, 410, 2556 Dondi, L., \& Ghisellini, G. 1995, MNRAS, 273, 583

Fomin, V. P., Stepanian, A. A., Lamb, R. C., et al. 1994, Astropart. Phys., 2, 137 Fruchter, A. J., Misra, K., Graham, J., et al. 2011, GCN, 11881

Gopal-Krishna, \& Wiita, P. J. 1992, A\&A, 259, 109

Graham, A. W. 2007, MNRAS, 379, $711 \mathrm{G}$

Halpern, J., Gezari, S., \& Komossa, S. 2004, ApJ, 604, 572

Hillas, A. M. 1985, Proc. of 19th ICRC, La Jolla, 445

Hills, J. G. 1975, Nature, 254, 295

Katarzyński, K., Ghisellini, G., Tavecchio, F., et al. 2005, A\&A, 433, 479

Komossa, S. 2002, In Rev. Mod. Astron., ed. R. E. Schielicke, 15, 27 (Wiley)

Leloudas, G., Malesani, D., Tanvir, N. R., et al. 2011, GCN, 11830

Leloudas, G., Malesani, D., Xu, D., et al. 2011, GCN, 11844

Levan, A. J., Tanvir, N. R., Wiersema, K., et al. 2011a, GCN, 11833

Levan, A. J., Perley, D., Tanvir, N. R., et al. 2011b, GCN, 11853

Levan, A. J., Tanvir, N R., Morgan, A., et al. 2011c, GCN, 11846

Li, T.-P., \& Ma, Y.-Q. 1983, ApJ, 272, 317

Lombardi, S., Berger, K., Colin, P., et al. 2011, Proc. of 32nd ICRC, Beijin, China [arXiv: 1109.6195]

Maksym, W. P., Ulmer, M. P., \& Eracleous, M. 2010, ApJ, 722, 1035
Mankuzhiyil, N., Ansoldi, S., Persic, M., \& Tavecchio, F. 2011, ApJ, 733, 14 Mattox, J. R., Bertsch, D. L., Chiang, J., et al. 1996, ApJ, 461, 396

Moralejo, A., Gaug, M., Carmona, E., et al. 2009, Proc. of 31st ICRC, Łódź, Poland [arXiv:0907.0943]

Moretti, E., Longo, F., Olivo, M., et al. 2009, in Societa' Italiana di Fisica Conference Proc., 102, The Shocking Universe - Gamma Ray Bursts and High Energy Shock Phenomena

Morgan, A. N., Bloom, J. S., Levan, A. J., et al. 2011, GCN, 11845

Myungshin, I., Urata, Y., Huang, K., et al. 2011, GCN, 11839

Omodei, N., Troja, E., Corbet, R., et al. (Fermi-LAT collaboration) 2011, GCN, 11862

Pittori, C., Verrecchia, F., Chen, A. W., et al. 2009, A\&A, 506, 1563

Rees, M. 1988, Nature, 333, 523

Rolke, W. A., López, A. M., \& Conrad, J. 2005, Nucl. Instrum. Meth., A551, 493

Sakamoto, T., Barthelmy, S. D., Baumgartner, W. H., et al. 2011, GCN, 11842 Svensson, R. 1987, MNRAS, 227, 403S

Tavani, M., Barbiellini, G., Argan, A., et al., for the AGILE Collaboration, 2009, A\&A, 502, 995

Thöne, C. C., Gorosabel, J., De Ugarte Postigo, A., et al. 2011, GCN, 11834

Zanin, R., Mazin, D., Carmona, E., et al. 2011, Proc. of 32nd ICRC, Beijin, China [arXiv: 1110.2987]

Zauderer, A., Berger, E., Frail, D. A., et al. 2011a, GCN, 11836

Zauderer, A., Berger, E., Frail, D. A., et al. 2011b, GCN, 11848

1 IFAE, Edifici Cn., Campus UAB, 08193 Bellaterra, Spain

2 INAF National Institute for Astrophysics, 00136 Rome, Italy

3 Università di Siena, and INFN Pisa, 53100 Siena, Italy

4 Universidad Complutense, 28040 Madrid, Spain

5 Technische Universität Dortmund, 44221 Dortmund, Germany

6 Max-Planck-Institut für Physik, 80805 München, Germany

7 Inst. de Astrofísica de Canarias, 38200 La Laguna, Tenerife, Spain

8 University of Łódź, 90236 Lodz, Poland

9 Depto. de Astrofísica, Universidad de La Laguna, 38206 La Laguna, Spain

10 Deutsches Elektronen-Synchrotron (DESY), 15738 Zeuthen, Germany

11 ETH Zurich, 8093 Zurich, Switzerland

12 Universität Würzburg, 97074 Würzburg, Germany

13 Centro de Investigaciones Energéticas, Medioambientales y Tecnológicas, 28040 Madrid, Spain

14 Università di Udine, and INFN Trieste, 33100 Udine, Italy

15 Inst. de Astrofísica de Andalucía (CSIC), 18080 Granada, Spain

16 Croatian MAGIC Consortium, Rudjer Boskovic Institute, University of Rijeka and University of Split, 10000 Zagreb, Croatia

17 Unitat de Física de les Radiacions, Departament de Física, and CERES-IEEC, Universitat Autònoma de Barcelona, 08193 Bellaterra, Spain

18 Università dell'Insubria, Como, 22100 Como, Italy

19 Institut de Ciències de l'Espai (IEEC-CSIC), 08193 Bellaterra, Spain

20 Tuorla Observatory, University of Turku, 21500 Piikkiö, Finland

21 Japanese MAGIC Consortium, Division of Physics and Astronomy, Kyoto University, Japan

22 Inst. for Nucl. Research and Nucl. Energy, 1784 Sofia, Bulgaria

23 Universitat de Barcelona (ICC/IEEC), 08028 Barcelona, Spain

24 Università di Padova and INFN, 35131 Padova, Italy

25 INAF/Osservatorio Astronomico and INFN, 34143 Trieste, Italy

26 Università di Pisa, and INFN Pisa, 56126 Pisa, Italy

27 ICREA, 08010 Barcelona, Spain

28 Now at École polytechnique fédérale de Lausanne (EPFL), Lausanne, Switzerland

29 Supported by INFN Padova

30 Now at: DESY, Zeuthen, Germany

31 Now at: Finnish Centre for Astronomy with ESO (FINCA), University of Turku, Finland

32 Also at Instituto de Fisica Teorica, UAM/CSIC, 28049 Madrid, Spain

33 Università di Trieste, and INFN Trieste, 34127 Trieste, Italy

34 Agenzia Spaziale Italiana (ASI) Science Data Center, 00044 Frascati, and INAF-Oar, 00040 Monteporzio Catone, Italy 